\title{
CONDICIONES TÉMPORO-ESPACIALES DE NIÑOS QUE HABITAN EN UNA COPROPIEDAD HORIZONTAL DE LA CIUDAD DE BARRAQUILLA, COLOMBIA
}

\section{TEMPORO-SPATIAL CONDITIONS OF CHILDREN LIVING IN HORIZONTAL CO-OWNERSHIP BUILDING AT BARRANOUILLA, COLOMBIA}

\begin{abstract}
Armando Aroca Araújo ${ }^{1}$, Lina Álvarez Toro ${ }^{2}$
${ }^{1}$ Doctorando en Educación énfasis en educación matemática. Universidad Francisco José de Caldas. Profesor Asociado. Universidad del Atlántico, calle 74 No. 38-100, Apto 901, Bloque 3, Conjunto Residencial Altos de Betania, Barranquilla, Colombia, e-mail: armandoaroca@mail.uniatlantico.edu.co; ${ }^{2}$ M.Sc. en Desarrollo infantil. Universidad de Manizales. Docente. Universidad del Atlántico, calle 74 No. 38-100, Apto 901, Bloque 3, Conjunto Residencial Altos de Betania, Barranquilla, Colombia, e-mail: linaalvarez@mail.uniatlantico.edu.co
\end{abstract}

Rev. U.D.C.A Act. \& Div. Cient. 19(2): 479-487, Julio-Diciembre, 2016

\section{RESUMEN}

La investigación tuvo como objetivo identificar las condiciones témporo-espaciales que tienen los niños, en un rango de edad de 8 a 11 años, residentes en una copropiedad horizontal en Barranquilla, Colombia, que registra tres bloques o edificios residenciales, una administración, áreas comunes, delimitación por paredes y un sistema de vigilancia. El problema que se identificó consiste en las condiciones de soledad, de confinamiento a la habitación, de adicción a los videojuegos, de sedentarismo y de pocas relaciones sociales que tienen los denominados niños de apartamento. La metodología empleada fue la de núcleos de educación social, NES. Los principales hallazgos tienen que ver con los seis espacios que emergieron en la investigación y sus respectivas temporalidades. Estos espacios son el externo, el limítrofe, el próximo, el local, el vertical y el subterráneo. La estrategia que se empleó, para poder identificar las condiciones témporo-espaciales, fue a través de la actividad de jugar y las expectativas que ellos tienen en la ampliación de su espacialidad. Se concluyó, que los niños, en el rango de edad seleccionado, tienen restricciones a áreas de juego, de esparcimiento y de problemas de interacción social con adolescentes.

Palabras clave: Núcleos de educación social, categorías, temporalidad, espacialidad.

\section{SUMMARY}

This research has as its main objective the identification of the temporal-spatial conditions of children aged 8 to 11, who are residents in a horizontal coparcenary in Barranquilla, Colombia, composed of three blocks or residential buildings, an administration, common areas and delimitated by walls and a monitoring system. The problem identified consists of the conditions of loneliness, confinement to the room, video game addiction, physical inactivity and poor social relationships that these "apartment children" have .The methodology used was the Nuclei of Social Education, NEF. The main findings have to do with the six spaces that emerged in the investigation and their respective time frames. These spaces are outside, the boundary, the nearby, the local, the vertical and the underground. The strategy was used to identify the temporal-spatial conditions through the activity of playing and the expectations these children have in expanding their spatiality. It was concluded that children in the age range selected have restrictions to play areas, spreading out and problems of social interaction with teenagers.

Key words: Nuclei of Social Education, Categories, temporality, spatiality.

\section{INTRODUCCIÓN}

En Colombia, se han adelantado algunas investigaciones relacionas con el objeto de estudio de este artículo, que más adelante se precisará. El grupo de investigación Convivencia y Ciudadanía de la escuela de trabajo social de la Universidad del Valle, ha desarrollado algunas de ellas, por ejemplo, Rincón \& Maldonado (2007) establecieron los motivos que conducen a las familias a vivir en este tipo de espacio. García \& Peralta (2015) han relacionado el espacio exterior con el lugar que toman las unidades residenciales. Otras fuentes 
sobre la copropiedad horizontal está en las normas que las rigen y promulgan, ley 675 de 2001 Congreso de la República de Colombia, diversos manuales y páginas Web, de empresas afines a lo inmobiliario. En el panorama internacional, se nota un poco más de fuentes bibliográficas sobre normas de convivencia, organización social o administración de la copropiedad horizontal, como los reportes de Cura (1993), Cerdá (1999) y Lasarte \& Feliu (2005).

Garrido (2005), al citar a Tuan (1983), sobre la manera que el niño va construyendo experiencia en el espacio geográfico, plantea lo siguiente: A medida que el niño crece, se va apegando a objetos, en lugar de apegarse a personas importantes, y finalmente a localidades. Para el niño, lugar es un tipo de objeto grande y un tanto inmóvil (...) El horizonte geográfico de un niño se expande, a medida que él crece, pero no necesariamente paso a paso en dirección a la escala mayor. Su interés y conocimiento se fijan, primero, en la pequeña comunidad local, después en la ciudad, saltando el barrio y de la ciudad, su interés se puede dirigir para la Nación y para lugares extranjeros, saltando la región.

En cuanto al tiempo y la temporalidad, para Vasco (2000) no se puede hablar mucho tiempo acerca del tiempo sin espacializarlo. Se trata de una metáfora obligada por nuestro cuerpo, por nuestras acciones y movimientos. Una primera acepción de la palabra "tiempo" es la temporalidad como propiedad inherente a todo proceso, relacionado con la dinamicidad; es aquello que permite decir que los procesos fluyen, duran, corren, discurren.

El problema que se abordó en esta investigación fueron las implicaciones que tienen las condiciones témporo-espaciales en los niños que habitan en copropiedad horizontal; para ello, se tuvo en cuenta, que el confinamiento al espacio local, donde el espacio colectivo y común se toma en estas unidades como todo lo que se encuentra de puertas para afuera de la residencia. Se construye para cumplir con un programa arquitectónico y se reduce a un mobiliario a utilizar por horas y según la edad, el género y la afición de los habitantes, pero no se proyecta como el lugar de socialización o de recreación.

En estos aspectos, también se encuentra el uso excesivo de video juegos, tal como lo señala Gómez et al. (2008), asociado con menores niveles de actividad física, aumentando el riesgo de desarrollar obesidad, debido al sedentarismo, tanto en la niñez como en la adultez. El análisis brinda elementos de juicio para concluir que se debe a la ausencia y el poco tiempo que sus padres pasan con sus hijos. Pérez \& Ruiz (2006) afirman que los hallazgos evidencian que la adicción a los video juegos cumple un papel dinámico, que pone en manifiesto una alteración que involucra, en su génesis, patrones de familia y entornos sociales inadecuados.
Basta, para ello, con hacer mención de lo que Cotton (1990) denomina los estresores psicosociales. Tal es el caso, por ejemplo, de aquellos individuos sometidos a condiciones de encierro y de hacinamiento: hogares con espacios minúsculos y con muchos miembros familiares, que impiden todo tipo de privacidad y libre circulación en el espacio disponible. El llamado efecto lata de sardinas puede resultar ser una experiencia traumática, dañina, estresante y generadora de altos y, a veces, incontenibles niveles de agresividad, como lo propone Lotito (2008).

Esta investigación es relevante, ya que el poder identificar las nociones témporo-espaciales vivenciadas por los niños que habitan en copropiedades horizontales permitirá reconocer cómo se desarrollan dichas nociones en relación a espacios delimitados por paredes, por señalizaciones y por restricciones de horarios en el uso de zonas de interacción, entre otros. Considerando la muestra seleccionada, los resultados no se podrán generalizar; sin embargo, estos son un antecedente para dicha temática.

\section{MATERIALES Y MÉTODOS}

La investigación, se llevó a cabo en una copropiedad horizontal de la ciudad de Barranquilla, Colombia, estrato 4. La unidad cuenta con tres bloques o edificios: dos, de 14 pisos y uno, de 15, con un total de 172 familias. Hay alrededor de 40 niños, desde recién nacidos hasta los 11 años. El número de adolescentes y jóvenes no es fácil de determinar, pues algunos, al parecer, comparten residencia de sus padres separados. En la unidad escogida cohabitan diversas familias: hombre con hombre, separadas con adolescentes o con jóvenes, parejas jóvenes sin hijos, adultas mayores solas, hombre con mujer y sus hijos jóvenes o adultos, entre otras opciones. La unidad tiene un pequeño parque, una cancha multifuncional de microfútbol y básquet y dos piscinas pequeñas: una para niños menores de cinco años y la otra para el resto de residentes. Tiene una casa club, que brinda los servicios de cine, de gimnasio y de billar. Cuenta con parqueaderos para visitantes y propietarios o inquilinos, más un sótano, que es exclusivo para parqueadero de vehículos de propietarios o inquilinos. La figura 1 representa un bosquejo de las once potenciales zonas horizontales de juego.

La investigación realizada es cualitativa, de acuerdo a Creswell (2007), de tipo etnográfico. El método empleado para este estudio fue la de núcleos de educación social, NES, de acuerdo con García \& Guerrero (2012), que se articulan en un proceso investigativo por etapas y escenarios de Investigación. En este proceso, se asumieron las etapas sujeto y copropiedad horizontal; por sujeto, se entiende los niños escogidos en el rango de edad establecido y por copropiedad horizontal, el lugar de investigación. El análisis de la información, se basó en el fichaje propuesta para los NES, que 


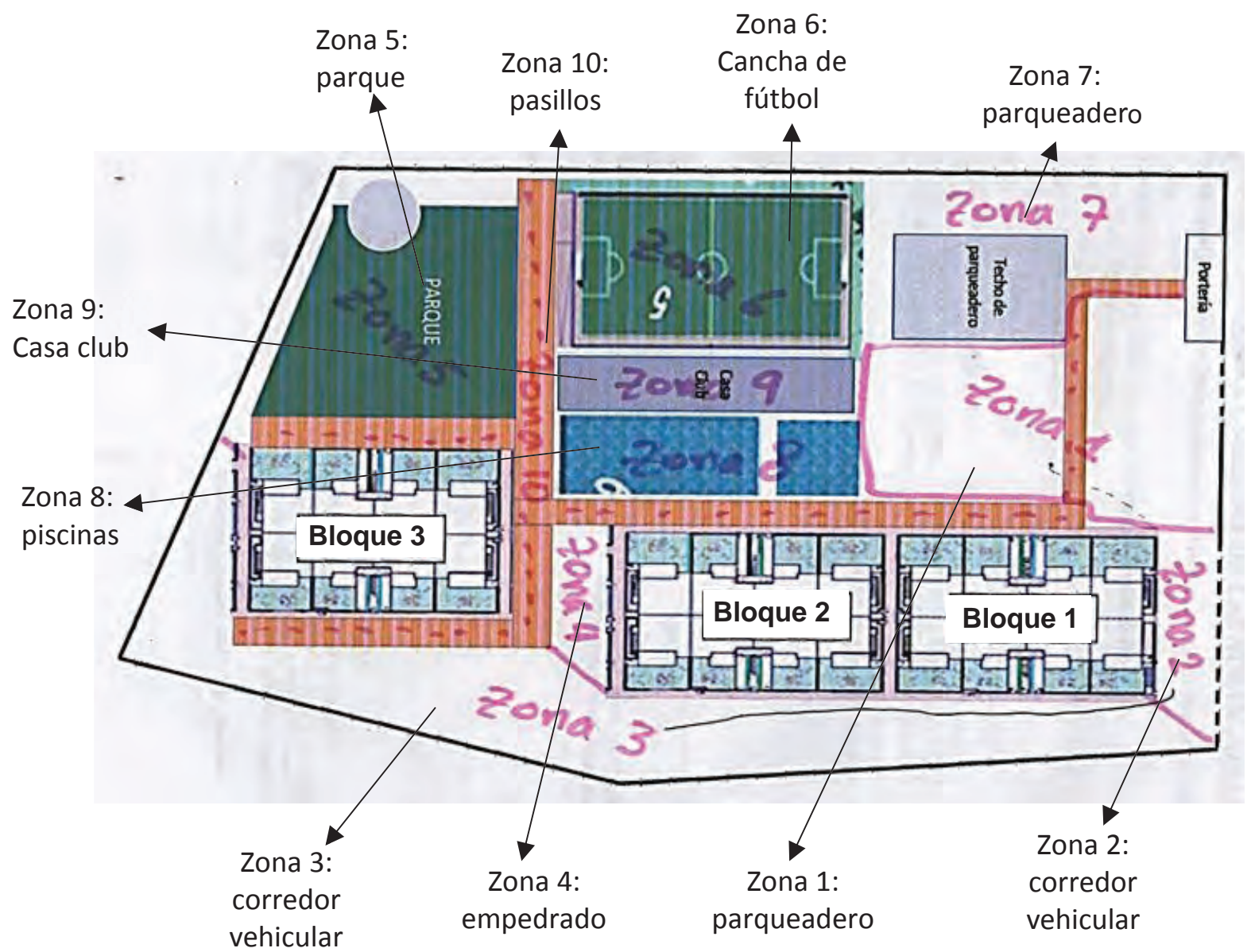

Figura 1. Las once zonas horizontales de juegos, establecidas en la Unidad residencial. Fotografía complementaria.

permitió identificar 27 categorías emergentes. Por medio de la actividad de jugar y con la información proporcionada por cuatro niños residentes en dicha unidad, se dividió en zonas potenciales de juegos: once horizontales y tres verticales, los bloques. Al momento de seleccionar los participantes en la unidad había diez niños y niñas. Los criterios de selección de la muestra fueron: el consentimiento de los padres, el asentimiento de los niños, el rango de edad y el tiempo de residencia en la unidad, no menor a un año. Por lo anterior, la muestra estuvo conformada por cuatro niños, de diez en total. Los cuatro niños que participaron tenían edades que oscilaban entre 8 a 11 años. Según Rael (2009), en estos niños la percepción del espacio se realiza a través del contacto con el entorno y les permite situarse en el espacio y reconocerlo, es decir, pueden reconocerse a sí mismos, reconocen su cuerpo, se pueden comunicar consigo mismo, con los demás, ponerse en relación con los objetos y con el espacio. La estrategia de conformación del grupo de participantes se hizo por medio de un grupo focal, de acuerdo con Mella (2000). En la sesión 1, de una hora y media, se empleó el grupo focal y las preguntas dirigidas a dicho grupo y las respuestas se grabaron; a continuación, se transcribieron literalmente y se hicieron las respectivas fichas de lectura, según el método NES. En la sesión 2, participaron tres niños; a ellos, se les pidió que respondieran por escrito algunas preguntas relacionadas con horarios de juegos, prohibiciones y prevenciones subjetivas o miedos a personas, cosas o animales; la figura 2 representa parte de la estrategia que se empleó para recoger información en las zonas verticales de juegos. Se les pidió a los niños que escribieran qué jugaban en cada uno de los pisos, a medida que íbamos bajando por las escaleras. 

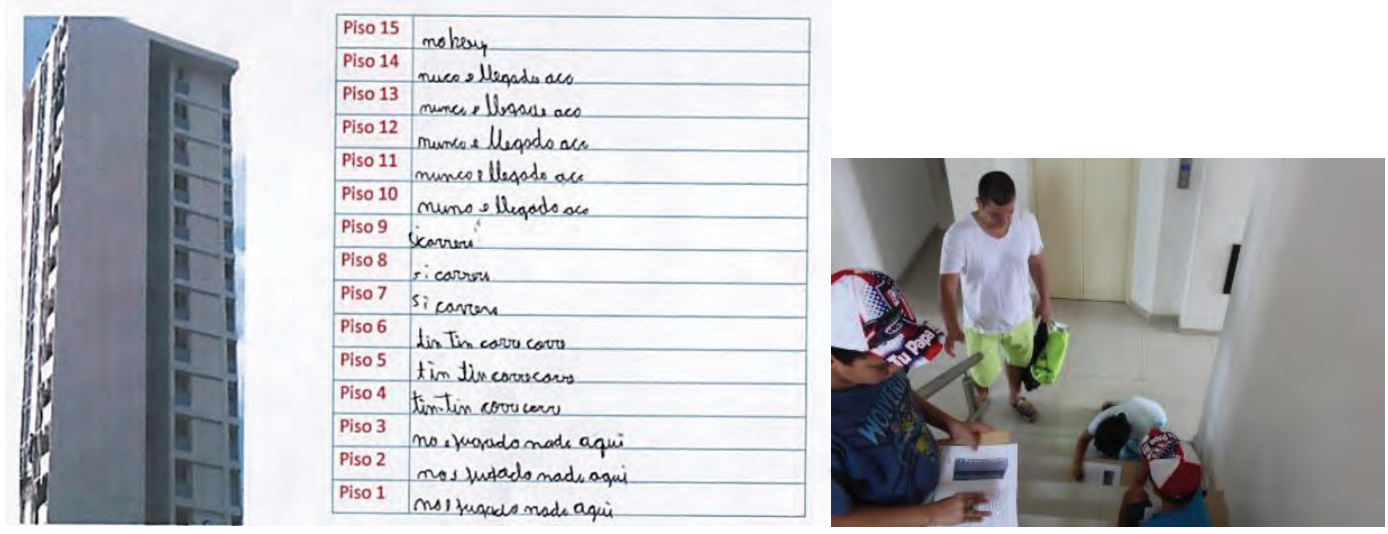

Figura 2. Formato para identificar los juegos que se desarrollan en zonas verticales de la copropiedad horizontal. Fotografía complementaria.

\section{RESULTADOS Y DISCUSIÓN}

La clasificación de los espacios que establecen condiciones témporo-espaciales a los niños residentes en la copropiedad horizontal ya descrita, a partir de las sesiones 1 y 2 , se describen en la tabla 1. En cada uno de estos espacios emergieron diversas categorías, basadas en las respuestas de los niños y que dan cuenta de sus nociones témporo-espaciales, según dicho espacio. A continuación, se hará una descripción de cada espacio y las categorías que emergieron en ellos.

Espacio limítrofe. La primera categoría que emergió fue Delimitaciones físicas del espacio periférico, que se sustenta en lo siguiente. A los niños, se les formularon las siguientes preguntas: ¿En la semana, de lunes a domingo, sales a jugar por fuera de la unidad?, ćcómo te sientes?, chay alguna diferencia de jugar dentro de la unidad y por fuera de ella? Las respuestas fueron las siguientes.
Niño 1: el domingo

Investigador: ¿Cómo te sientes tú, cuando sales a jugar por fuera de la unidad?

Niño 1: libre

Investigador: ¿Y cuando juegas aquí adentro cómo te sientes?

Niño 1: encerrado

El espacio limítrofe de la copropiedad horizontal, se establece por la principal barrera observable, la pared: siendo la principal delimitación física, un obstáculo para lo que los niños denominan libertad. Más adelante, se profundizará en ello y lo que implica este tipo de encerramiento, con la sensación de seguridad.

Las segunda categoría que emergió fue Reconocimiento administrativo, familiar o social y ampliación de la espacialidad: lo que dio origen a esta investigación fue el capta huellas o huellero, que es un dispositivo electrónico, donde los resi-

Tabla 1. Clasificación de los espacios que establecen condiciones témporo-espaciales a los niños.

\begin{tabular}{|c|c|c|}
\hline \multirow{6}{*}{ 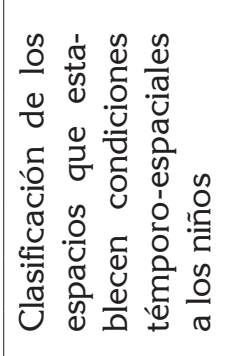 } & Limítrofe & $\begin{array}{l}\text { Referido a las fronteras, en este caso, tiene paredes que la delimitan con el resto del } \\
\text { barrio }\end{array}$ \\
\hline & Próximo & Referido al espacio interno, que juega una especie de extensión del espacio local \\
\hline & Local & Se trata del espacio familiar, el del apartamento \\
\hline & Vertical & Son los bloques o edificios \\
\hline & Subterráneo & Parqueadero de carros y motos en el nivel -1 del ascensor \\
\hline & Externo & Correspondiente a lo externo, el barrio \\
\hline
\end{tabular}


dentes autorizados colocan su huella digital y la puerta, que da al espacio exterior, se abre de inmediato; los niños desean tener la huella registrada y poder salir de la copropiedad horizontal. Para los niños salir a la tienda de la esquina, solos, significa ampliar su espacialidad.

Dentro de este reconocimiento, se evidenció una tercera categoría, que se denominó la edad, como condición subjetiva en la ampliación de la espacialidad: al preguntarles si les gustaría tener la huella registrada, todos respondieron que sí. Luego se les preguntó ca los cuántos años creen ustedes que los van a dejar salir solos? A partir de los quinces o dieciocho, respondieron. Los niños tienen en su mente una edad que les permitirá ampliar su temporalidad y espacialidad, a otros límites diferentes de la tienda de la esquina y del parque más cercano. Se trata de un rango de edad, donde, según ellos, alcanzan la adultez, la independencia. Tai et al. (2006) refieren que los niños anhelan esa adultez, ya que en la niñez no tienen la libertad de explorar libremente el ambiente. La edad, entonces, juega un papel en la configuración de los espacios y del tiempo y de la misma experiencia socio-geográfica de los niños.

La cuarta categoría emergente del espacio limítrofe es el concepto de Unidad residencial en el recorrido mental: a los niños se les pidió que hicieran un recorrido mental por la copropiedad horizontal, desde el punto donde se realizó la sesión 1. ¿Qué llamó la atención en estas respuestas? Que el recorrido de niño 3 es distinto a los de niño1, niño 2 y niño 4. El niño 3 incluyó los pasillos, cuando dice "pasé por el bloque 2 el bloque 3". Los otros niños, que poco salen al parque Betania, la copropiedad horizontal es el contorno de la unidad; se fueron bordeando la pared. Se considera que esta diferencia está en que el niño 3 tiene mayor experiencia socio-geográfica y no por su edad.

La quinta categoría son lugares de mayor gusto: aparecen la cancha de fútbol, la piscina y el cine. Estos espacios de juego son los de mayor gusto, porque se comparte con otros, con amigos o familiares. La que tuvo mayor frecuencia en el gusto fue la cancha de fútbol; sin embargo, este es uno de los lugares de más conflictos que estos niños tienen en la copropiedad horizontal, debido a los desplazamientos que sufren, regularmente, por otros niños de otro rango de edad mayor, que ellos denominaron los adolescentes, "nos quitan la cancha". De esta forma, se configuró la categoría Delimitaciones forzosas de espacio de juego por interacción con otros rangos de edad. Según Calvo et al. (2001), Sánchez et al. (2006), Garaigordobil (2009), Mestre et al. (2009), se registró un incremento en la empatía durante la adolescencia, pero únicamente en las chicas. Una posible explicación de este resultado, se puede deber a que las mujeres, en todas las edades, alcanzan un mayor nivel de conductas de resolución positivas de conflictos, mientras que los chicos tienen mayores niveles de resolución agresiva de conflictos, tal como lo señala Garaigordobil \& Maganto (2011).

Espacio próximo. En este espacio próximo emergieron dos categorías, que se complementan: el Espacio próximo, como extensión del espacio local y Confort, en el espacio próximo y local. Cuando se les preguntó a los niños si les gustaría vivir en una casa o en otra copropiedad horizontal o si se sienten bien viviendo en la actual, se pudo reconocer dos momentos en las respuestas. Las interpretaciones de los niños, aparentemente, son contradictorias, porque en diversas respuestas en la sesión 1 manifiestan su aburrimiento, soledad o angustia y al preguntárseles si deseaban vivir en otra unidad, los niños 1 y 3 manifiestan que se siente bien al vivir en la suya; sin embargo, el tema de fondo es la seguridad, en particular, cuando se nombra al portero y a las cámaras de seguridad en las esquinas de la copropiedad horizontal, en los ascensores y en las zonas de juego y, además, las paredes, que le dan un lugar a la unidad con el resto del barrio. De hecho, el niño 3 manifestó que antes de vivir en la copropiedad horizontal lo hacía en una casa, donde fueron atracados. El niño 4 llegó a manifestar que él no se mudaría para una casa.

Los conjuntos de vivienda de acceso controlado son expresiones de la tendencia al encerramiento de los urbanistas en aras de protegerse de los hechos de violencia y de delincuencia. Además, en el transcurso de la historia, los pueblos cercados con muros fueron diseñados para proteger a los habitantes y sus propiedades, de acuerdo con García \& Peralta (2015). Según González (2007), los espacios cerrados se constituyen en una respuesta social a la necesidad de protección frente a aquellos considerados extraños, quienes representan una amenaza a la existencia individual. Para Bauman (2005), se está hablando de una inseguridad subjetiva que nace de la mixofobia. López \& Rodríguez (2005) consideran que es aprovechado por los planificadores urbanos, para diseñar propuestas habitacionales, que seudosatisfacen las demandas de los ciudadanos.

El tema de la seguridad permea otras categorías, como las Condiciones de acceso al entorno próximo: salir y entrar del apartamento es posible gracias a cierta confianza de los padres en la seguridad que ofrece la copropiedad horizontal; sin embargo, siempre están algunas prevenciones, como no entrar a otros apartamentos, no conversar con adultos, entre otras. Girola (2005) amplía el panorama de cómo el trasladarse a una copropiedad horizontal responde a una decisión familiar, que contempla varios factores: la trayectoria residencial, la historia personal, el lugar anterior de residencia, la situación socioeconómica, las oportunidades laborales, el deseo de criar a los hijos en ambientes más seguros.

En este espacio, se encontró una nueva categoría titulada Transgresión de la normativa del espacio próximo: la copro- 
piedad horizontal tiene espacios reglamentados para su uso. Los baños que se encuentran en el área de la piscina hacen parte de las zonas en las que está establecido no ingresar después de las 6:00 p.m.; sin embargo, este lugar es utilizado por los niños para jugar escondite. Esta transgresión al reglamento, se debe a que las zonas de juego no son suficientes; sea por horarios, preferencia a niños menores para uso del parque, restricciones o áreas disponibles para el juego. La necesidad de jugar en estas condiciones tan delimitadas y restringidas implica transgredir normas temporales y espaciales.

A continuación, se presentan otras tres categorías, que emergieron en el espacio próximo. 1) Número de amigos y posibilidades de jugar: más amigos implica más posibilidades de jugar cuando se sale del espacio local al espacio próximo. Más amigos tienen implicaciones de orden temporal.

2) Espacio próximo restringido por temores personales: el espacio también se configura por temores personales o subjetivamente. Estos temores estaban relacionados con personas, familias, mascotas o zonas oscuras, es decir, responde a un orden de interacción y de experiencias con el medio. Los niños y los mismos adultos evitan pasar por cierto pasillo o apartamento, para no verse con una persona, una mascota u otra situación.

3) Diversos recorridos espaciales y cálculo de distancias: esta categoría emerge de las respuesta a: usted está en su cama y se van a imaginar cuál es el lugar o punto más lejos que hay desde su cama, pero que este aquí adentro de la unidad. Algunas respuestas fueron las siguientes.

Niño 4: porque... o sea, la portería, yo estoy en el bloque 3 tengo que bajar el ascensor y la portería es como el final de la unidad, porque la portería si tú ya sigues caminando derecho, ahí ya te saldrías de la unidad.

Niño 3: que a él se le haría más difícil ir para allá y más difícil ir para allá y a él se le haría más fácil, porque a él se le haría más fácil haciendo fi fu fa

Investigador: co sea fi fu fa es qué? ...el recorrido por los pasillos, por eso cuando tú estás haciendo pasillos, los pasillos. Niño 3: no, por ejemplo, él sale de su casa va para allá y llega a la portería, se le hace refácil que yo a la portería y no es lo mismo. Y yo dije que no era igual.

No es lo mismo la distancia más corta o más lejana de un niño que vive en un piso 9 que de un niño que vive en el primer piso, como sucede con niño 4 y niño 1; por ejemplo, el niño 1 , su punto más lejano de su cama es la parte de atrás del bloque 3, mientras que el punto más lejano para el niño 4 es la portería, debido a que él debe recorrer nueve pisos para llegar hasta dicho punto. Así, el tiempo y el espacio recorrido para un niño que vive en apartamentos altos, no es el mismo para aquellos que viven en el piso 1 .
Espacio local. La principal categoría en este espacio es Temporalidad semanal: esta categoría emerge al formularles dos preguntas a los niños chay algún día o varios días de la semana en el que te sientas aburrido?, ċcuál y por qué? y chay algún día o varios días en que no estés aburrido? Las actividades escolares marcan condiciones en la temporalidad de los niños que, incluso, no es propia de residentes en copropiedades. Se pudo identificar que hay cuatro comportamientos relacionado a la temporalidad semanal. Estos se reflejan en lo que piensan los niños sobre el lunes, el periodo entre lunes y jueves, el viernes y el fin de semana o el puente. El periodo lunes a jueves es asociado a compromisos escolares y más restricciones para jugar; el viernes, sienten cierto placer y descanso; el fin de semana hay más libertad para hacer, sin restricción, algunas de las cosas que les gusta hacer; significa compartir con los padres de familia o la familia en otros espacios. También hay libertad para acostarse tarde sin límite alguno y hay libertad para jugar todas las horas deseadas en video juegos. Lo que es característico en este caso es que entre el lunes a jueves hay mayor permanencia en el espacio local.

Las relaciones entre niños y horarios laborales de los padres de familia implicaron que emergieran tres categorías, que se describen a continuación. 1) Soledad, temor y espacio local: hay diversos factores que reducen la movilidad del niño al espacio local, entre ellos, las ocupaciones laborales de los padres. Los conflictos para conciliar trabajo-familia, se presentan cuando las presiones del medio hacen que trabajo y familia no sean compatibles, en algún momento, para lo anterior. Yang et al. (2000) establecieron que existen ciertos tipos de conflictos al respecto: Uno, es el que se da cuando el tiempo utilizado en una de las dos funciones impide destinar tiempo a la otra y el segundo ocurre cuando se dan altos niveles de tensión y carga en el cumplimiento de las actividades en uno de estos ámbitos, lo que, a su vez, afecta el desempeño en el otro, por lo tanto, no existe una instancia que dirima sobre el equilibrio entre el tiempo reservado al trabajo y el consagrado a la educación de los hijos y a la atención a la familia, según lo planteado por Arriagada (2005). 2) Soledad momentánea y espacio local: el niño 4 manifestó que su espacio local no se ve afectado, mientras queda sin compañía, cuando su madre o padre sale a la portería a buscar alguna encomienda o recibo, es decir, mientras el periodo de soledad es corto y que el niño tenga una estimación de este tiempo, su espacio local no se verá afectado por sus temores. 3) Acompañamiento familiar y espacio local: si el niño tiene hermanos mayores, juegan un papel esencial en brindar seguridad a los hermanos menores, cuando los padres no están presentes. El espacio de juego no restringe solo al espacio local, sino al espacio interno de la copropiedad horizontal. 
Espacio vertical o zonas verticales de juego. En este espacio, se encontró una primera categoría, que se denominó Configuración del espacio vertical para zonas de juegos: ante las delimitaciones horizontales del espacio para zonas de juego y los conflictos que se dan en las establecidas, los niños de este rango de edad han optado por convertir las escaleras y pisos de los bloques en una alternativa de zona de juego. El rin rin corre corre es uno de los juegos preferido y peligroso de los niños; se trata de tocar los cuatro timbres de los apartamentos de un mismo piso y salir corriendo hacia abajo por las escaleras.

La configuración del espacio vertical en zonas recreacionales evidenció diferentes juegos, que son característicos en una copropiedad horizontal, cuyos edificios tienen escalera y ascensor: Carrera, sea bajando corriendo por las escaleras o persiguiendo un balón que baja por gravedad, un juego de gravedad; Tin tin corre; con la patineta en los pasillos; el escondite, pero en pisos bajos e ir presionando el botón del ascensor en cada piso y bajar corriendo por las escaleras, este juego molesta mucho a los adultos.

Una segunda categoría hace referencia a Pisos altos y bajos y configuración témporo-espacial del entorno próximo: las condiciones témporo-espaciales para niños que viven en un primer piso son distintas para aquellos que viven en pisos más altos. Los bloques 1 y 2 limitan con zonas de parqueo y una calle donde los carros transitan regularmente; los padres de estos niños los previenen continuamente, mientras tanto los padres de los niños de pisos bajos del bloque 3 no tienen esta preocupación, porque dicho bloque limita con el parque y por detrás está la parte terminal de la calle.

Una tercerea categoría en este espacio es la Configuración de espacios verticales de juegos por presunta evasión de res- ponsabilidad: hay una tendencia de los niños a jugar más en otros bloques donde ellos no residen y, por lo general, en el más distante de su espacio local. Los niños configuran, espacialmente, los bloques como zonas verticales de juego, a partir de una evasión de responsabilidad, por la posibilidad de cometer algún error o violación del reglamento interno o de las advertencias de los padres.

El espacio subterráneo. Se encontró una única categoría en este espacio, Recorrido mental por el sótano o parqueadero subterráneo: a pesar que transitar por el sótano puede implicar un grave accidente con un automóvil o una motocicleta y que se presentan temores que emergen por la oscuridad y las picaduras de insectos, los niños transitan por él, como se pudo comprobar en la sesión 2 . Los niños, se restringen el paso por el sótano, no por los letreros que ha colocado la administración de prohibido jugar, sino por los temores que les genera; sin embargo, el sótano se convierte en un corredor estratégico de comunicación interbloques cuando está lloviendo. En una copropiedad horizontal, la lluvia restringe unos espacios, pero habilita otros. La figura 3 muestra una ruta que hizo niño 4 que, regularmente, usan todos los niños para poderse comunicar entre sí y, en algunos casos, jugar.

El espacio externo. De la sesión 1, emergieron algunas categorías para el espacio externo y se describen a continuación.

Nuevas experiencias y ampliación de la espacialidad: una nueva experiencia no es lo mismo a sentirse libre, pues estar encerrado en otro espacio es una nueva experiencia; una nueva experiencia, en este caso, implica libertad para conocer otros lugares. Felicidad y ampliación de la espacialidad: sentirse feliz para los niños, en este tema de investigación, significa poder salir del encierro rutinario del espacio local.

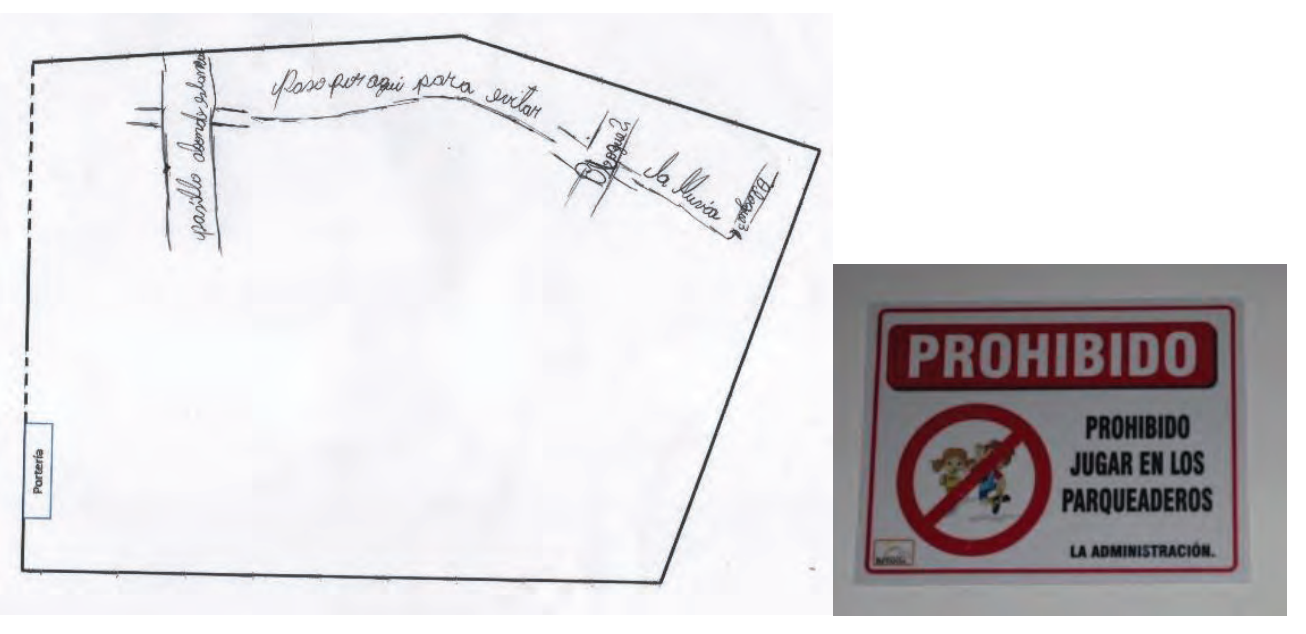

Figura 3. Ruta de comunicación interbloques mientras llueve. Fotografía complementaria. 
Atraco y rapto, seguridad personal, en la ampliación de la espacialidad: aquí, se dan tres subcategorías: dos explícitas, que son atraco y rapto, en la ampliación de la espacialidad, pero ambas están interconectadas por una categoría implícita, referida a la seguridad personal, en la ampliación de la espacialidad; ambos sentimientos de atraco y de rapto, cuando los niños se imaginaron salir solos al espacio externo, se dan tanto a priori como a posteriori.

Expectativas en la ampliación de la espacialidad: el tener acceso a otro lugar diferente a la copropiedad residencial abre un abanico de posibilidades; los niños saben que al salir de dicho espacio pueden acceder a juegos, comidas, lugares y actividades, que los llenarían de alegría y de libertad y estas expectativas son más fuertes que la inseguridad que sienten o presienten al salir solos de la unidad. La superación a esos temores son vencidos por satisfacciones personales que hacen parte de la vida social.

La familia en la ampliación de la espacialidad: en conversación con algunas madres de familia recuerdan que en su niñez o juventud los padres de familia lo que hacían era salir a la calle a buscar a sus hijos, muy poco existían las unidades residenciales, eran casas y los niños jugaban felices y sin preocupaciones en las calles. Ahora, no; se trata, en estas condiciones de vida, de enviar a los niños al espacio próximo, a que jueguen, a que eviten entretenerse con juegos electrónicos y dejen de estar varias horas frente a un computador o un video juego.

Para finalizar, los niños manifiestan el interés por ampliar su espacialidad, pasando de un espacio limítrofe a un espacio externo, donde ellos ven una posible libertad hacia la exploración de una nueva experiencia, libertad y felicidad. Reconocen el encerramiento, la portería y las entradas vehiculares que rodean la Unidad residencial, que se denominó espacio limítrofe, como un obstáculo para el desarrollo de la libertad y ampliación de su espacialidad y temporalidad; no obstante, confían en ese espacio limítrofe, como representación de su seguridad propia y de su familia. El espacio próximo, que es en parte extensión del espacio local en una copropiedad horizontal, tiene pocas zonas de juego y en cambio diversas restricciones normativas o sociales que obligan a los niños a transgredir las normas, tanto temporales como espaciales, para ellos poder jugar. Para cerrar, un llamado a aquellas administraciones de unidades residenciales que solo se dedican a administrar el terreno y los demás bienes comunes, no es solo eso sino también se trata de contribuir al bienestar de los niños y depende de las condiciones sociales de cada copropiedad horizontal y del interés de los padres de familia por tomar la iniciativa.

Conflictos de intereses: El manuscrito fue preparado y revisado con la participación de todos los autores, quienes de- claramos que no existe conflicto de intereses que ponga en riesgo la validez de los resultados presentados.

\section{BIBLIOGRAFÍA}

1. ARRIAGADA, I. 2005. Los límites del uso del tiempo: dificultades para las políticas de conciliación familia y trabajo. Políticas hacia las familias, protección e inclusión social, CEPAL-UNFPA. Serie de Seminarios y Conferencias, 46. Disponible desde Internet en: http://repositorio.cepal.org/handle/11362/6808 (con acceso 01/06/2016).

2. BAUMAN, Z. 2005. Amor líquido. Acerca de la fragilidad de los vínculos humanos. Fondo de Cultura Económica de Argentina. 203p.

3. CALVO, A.; GONZALEZ, R.; MARTORELL, M. 2001. Variables relacionadas con la conducta prosocial en la infancia y adolescencia; personalidad, autoconcepto y género. Infancia y aprendizaje: J. Study Educ. Developm. 2(1):95-111.

4. CERDÁ, R. 1999. Manual de propiedad horizontal y arrendamientos. Disponible desde Internet en: http:// www.sociedadesurgentes.com/arrendamientos.pdf (con acceso 03/06/2016).

5. CONGRESO DE LA REPÚBLICA DE COLOMBIA. 2001. Ley 675. Disponible desde Internet en http:// www.alcaldiabogota.gov.co/sisjur/normas/Norma1. jsp? $\mathrm{i}=4162$ (con acceso 29/05/2016).

6. COTTON, D. 1990. Stress Management: An integrated approach to therapy. New York. Psychology Press, 264p.

7. CRESWELL, J.W. 2007. Qualitative inquiry and research design. Choosing among five traditions. Thousand Oaks, CA: Sage Publications, Inc. 395p.

8. CURA, D.C. 1993. Propiedad horizontal y condominio. Ejercicio de los derechos. Revista la Ley (Argentina). 1(153):2.

9. GARAIGORDOBIL, M. 2009. A comparative analysis of empathy in childhood and adolescence: Gender differences an associated socio-emotional variables. Int. J. Psycholog. Therapy (España). 9(2):217-235.

10. GARAIGORDOBIL, M.; MAGANTO, C. 2011. Empatía y resolución de conflictos durante la infancia y adolescencia. Rev. Latinoam. Psicología. (Colombia). 23(2):255-256. 
11. GARCÍA, F.A.; PERALTA, M.D. 2015. Urbanizaciones cerradas y su vinculación con el espacio exterior en la ciudad de Cali: una propuesta metodológica para su análisis. Prospectiva (Colombia). 19:197-221.

12. GARCÍA, B.Y.; GUERRERO, J. 2012. Núcleos de Educación Social - NES. Investigación, Prevención y Participación. Universidad Distrital Francisco José de Caldas (Colombia). 144p.

13. GARRIDO, M. 2005. El espacio por aprender, el mismo que enseñar: las urgencias de la educación geográfica. Cad. Cedes (Brasil). 25(66):137-163.

14. GIROLA, M. 2005. Tendencias globales, procesos locales: una aproximación al Fenómeno de los conjuntos residenciales con seguridad de la región metropolitana de Buenos Aires. AIBR. Rev. Antropol. Iberoam. (España). 43:1-24.

15. GÓMEZ, L.F.; LUCUMI, D.I.; PARRA, D.C.; LOBELO, F. 2008. Niveles de urbanización, uso de televisión y videojuegos en niños colombianos posibles implicaciones en Salud Publica. Rev. Salud Pública. 10(4):505-516.

16. GONZÁLEZ, E. 2007. Biografía del miedo. Los temores en la sociedad contemporánea. Random House Mondadori (Barcelona). 320p.

17. LASARTE, C.; FELIU, M.I. 2005. Comunidades de vecinos y administración de fincas urbanas y complejos residenciales. TECNOS (España). 1026p.

18. LÓPEZ, L.; RODRÍGUEZ, I. 2005. Evidencias y discursos del miedo en la ciudad: casos mexicanos. SCRIPTA NOVA. Revista electrónica de geografía y ciencias sociales (España). IX. 194 (54). Disponible desde Internet en: http://www.ub.edu/geocrit/sn/sn-194-54. htm (con acceso 01/06/2016).

19. LOTITO, F. 2008. Estrés: el azote del siglo XXI. Un trastorno transversal que afecta a niños, adolescentes y adultos. Santiago: Editorial Puerto de Palos.

20. MELLA, O. 2000. Grupos Focales (“Focus Groups”). Técnica de investigación cualitativa. Documento de tra- bajo No. 3, Cide, Santiago, Chile. Disponible desde Internet en: http://biblioteca.uahurtado.cl/ujah/856/ txtcompleto/txt105091.pdf (con acceso 04/022016).

21. MESTRE, M.; SAMPER, P.; FRIAS, M.; TUR, A. 2009. Are women more empathetic tan men a longitudinal study in adolescence. The Spanish J. Psychology. 12(1):6-83.

22. PÉREZ M., J.; RUIZ, J.I. 2006. Influencia del video juego en la conducta y habilidades que desarrolllan los video jugadores. Rev. Electr. Tecnologia Educativa. 21:14p.

23. RAEL, I. 2009. Espacio y tiempo en educación infantil. Revista innovación y experiencias educativas. 1(15):1-11.

24. RINCÓN, M.T.; MALDONADO, M.C. 2007. Acerca de los motivos para vivir en espacios residenciales multifamiliares. Prospectiva. 12:147-180.

25. SÁNCHEZ, Q.; OLIVA, A.; PARRA, A. 2006. Empatía y conducta prosocial durante la adolescencia. Rev. Psicología Social. 21(3):259-271.

26. TAI, L.; KNIGHT, E.; MCLELLAN, G.; HAQUE, M. 2006. Designing outdoor Environments for children. Landscaping Schoolyards, Gardens, and Playgrunds. McGraw-Hill (New York). 380p.

27. VASCO, C. 2000. El tiempo en la teoría general de procesos y sistemas. En: Lopera, J. (ed.). El problema del tiempo. Universidad nacional de Colombia, Sede Medellín (Medellín). p.215-240.

28. YANG, N.; CHEN, C.C.; CHOI, J.; ZOU, Y. 2000. Sources of work-family conflict: a sino-U.S. comparison of the effects of work and family demands. Acad. Managem. J. 43:113-123.

Recibido: Junio 10 de 2016

Aceptado: Noviembre 1 de 2016

\section{Cómo citar:}

Aroca Araújo, A.; Álvarez Toro, L. 2016. Condiciones témporo-espaciales de niños que habitan en una copropiedad horizontal de la ciudad de Barraquilla, Colombia. Rev. U.D.C.A Act. \& Div. Cient. 19(2): 479-487. 